\title{
Flow and heat transfer characteristics of blooming jets impinging upon wall using DNS
}

\author{
Kentaro ECHIGO*, Koichi TSUJIMOTO*, \\ Toshihiko SHAKOUCHI* and Toshitake ANDO* \\ *Department of Mechanical Engineering, Mie University \\ 1577 Kurimamachiya-cho, Tsu 514-8507, Japan \\ E-mail: echigo@ees.mach.mie-u.ac.jp
}

Received: 17 November 2019; Revised: 25 February 2020; Accepted: 19 March 2020

\begin{abstract}
A single impinging jet exhibits high heat transfer performance around an impingement point on a wall. However, the heat transfer performance deteriorates as it moves away from the impingement point. Consequently, multiple impinging jets are commonly introduced to overcome the shortcomings of a single jet: inhomogeneous heat distribution on the wall and a narrow heating area. However, inhomogeneous heat transfers still occur. Therefore, a new jet control is required to improve the uniformity of heat transfer. Meanwhile, blooming jets are produced by appropriate combinations of axial and helical excitations at the nozzle exit. Using appropriately selected excitations, a jet can split into two separate jets (bifurcating jet) or spread into a shower of toroidal vortex rings. Blooming jets exhibit good performances of mixing and diffusion, suggesting possible applications in flow control. However, studies regarding the heat transfer performance of blooming jets are non-existent. In this study, we conducted direct numerical simulations of blooming jets impinging upon a wall and investigated their flow characteristics and heat transfer performances. As control parameters, the impingement distance (the distance from the nozzle to the wall) and frequency ratio (the axial excitation frequency to the helical frequency) are varied. The vortex structures and velocity magnitude reveals flow modulations due to blooming control. With the time-averaged local Nusselt number, the heat transfer performance of the blooming jets is evaluated quantitatively. Compared with uncontrolled jets, the uniformity of heat transfer of blooming jets is better, suggesting their potential application for leveling the heat transfer of impinging jets.
\end{abstract}

Keywords : Direct numerical simulation (DNS), Blooming jet, Flow control, Impinging jet, Heat transfer

\section{Introduction}

Impinging jets are widely used in cooling industrial applications, such as electric devices, blades of gas turbines, and hot steel, owing to its high heat transfer performance and easy implementation into various systems. These characteristics have been reviewed previously (Kataoka, 1990) (Viskanta, 1993) (Jambunathan et al., 1992). An impinging jet can be subdivided into three regions, i.e., the free jet, stagnation, and wall jet regions; the heat transfer performance on a wall depends primarily on the Reynolds number, nozzle shape, number of nozzles, and distance from the nozzle to the impingement wall (impingement distance). A single impinging jet produces a high heat transfer rate around the impingement point on the impingement wall. However, the heat transfer performance deteriorates as it moves away from the impingement point. Hence, to overcome the shortcomings of a single impinging jet, such as the inhomogeneous heat distributions on the wall and narrow heating areas, appropriate control methods should be introduced in various industrial applications.

Recently, significant advances in computing power have resulted in the realization of fluid phenomena, including miniature vortices, using direct numerical simulation (DNS). Furthermore, we discovered that the active control of a 
round jet is effective for mixing enhancement (Tsujimoto et al., 2008). The DNSs of impinging jets (Satake et al., 1998) (Tsubokura et al., 2003) (Hattori and Nagano, 2004) (Dairay et al., 2015) and highly resolved LESs (large eddy simulations) have demonstrated the flow structure and statistical heat transfer properties of impinging jets (Hadžiabdić and Hanjalić, 2007) (Dairay et al., 2014), thereby enabling the systematic investigation of, the effects of control parameters.

To enhance mixing and diffusion in industrial applications, the mixing control of jets has been examined. The control methods used for jet mixing can be categorized into either passive or active methods. The results of linear stability analyses revealed two types of dominant modes characterizing the large-scale flow structures in the near field of the jet: varicose and helical modes, in which diffusion and mixing can be controlled effectively. Furthermore, a complex jet is produced by a combination of these modes (Lee and Reynolds, 1985) (Reynolds et al., 2003). For example, a flapping mode comprises a pair of helical modes with the same frequency and same amplitude. Moreover, it has been experimentally confirmed that when the axial mode is added to them, a bifurcating jet or a blooming jet is formed (Lee and Reynolds, 1985) (Reynolds et al., 2003). Such an active control, which generated strong diffusions, has also been investigated using DNS (Freund and Moin, 2000) (Danaila and Boersma, 2000) (Hilgers and Boersma, 2001) (Gohil et al., 2010) (Gohil et al., 2015) and LES (Silva and Metais, 2002) (Tyliszczak and Boguslawski, 2007) (Tyliszczak and Geurts, 2014) (Tyliszczak and Geurts, 2015).

Recently, blooming jets have been studied by (Gohil et al., 2015) and (Tyliszczak, 2015) (Tyliszczak, 2018) using DNS, which yielded the detailed frequency characteristics of vortical structures and mean flow properties. However, the performances of blooming jets have been examined for free jets only hitherto; they have never been applied for impinging jets, to our best knowledge.

In the present study, to improve the uniformity of heat transfer on an impingement wall, we propose a control method in which a blooming round jet is arranged at an inflow. As control parameters, the frequency ratio of the helical and axial modes of the blooming jet and the impingement distance were varied. Furthermore, to accurately predict the heat transfer performance of the proposed method, we conducted DNS, in which a sine or cosine series and a sixth-order compact scheme were assembled, and a fringe technique (Nordstrom et al., 1999) was used to realize a surrounding boundary condition. From the perspective of time-averaged physical quantities, it was discovered that the Nusselt number on the impingement wall was better than that of the uncontrolled case.

\section{Numerical method}

\subsection{Governing equations and discretization}

Under the assumption of incompressible flows, the governing equations are as follows:

$$
\begin{aligned}
& \frac{\partial u_{i}}{\partial x_{i}}=0 \\
& \frac{\partial u_{i}}{\partial t}+h_{i}=-\frac{\partial p}{\partial x_{i}}+\frac{1}{\operatorname{Re}} \frac{\partial^{2} u_{i}}{\partial x_{i} \partial x_{i}} \quad\left(h_{i}=\varepsilon_{i j k} \omega_{j} u_{k} \omega_{j}: \text { vorticity }\right) \\
& \frac{\partial T}{\partial t}+u_{i} \frac{\partial T}{\partial x_{i}}=\frac{1}{\operatorname{RePr}} \frac{\partial^{2} T}{\partial x_{i} \partial x_{i}},
\end{aligned}
$$

where the convective terms in Eq. (2) is expressed in the rotational form to conserve the total energy; therefore, $p$ represents the total pressure (Canuto et al., 1988). The equations above are normalized by the diameter of the inlet jet, $D$ and the inlet velocity, $V_{0}$. The Reynolds number and Prandtl number are defined as $\operatorname{Re}=V_{0} D / v(v:$ dynamic viscosity) and $\operatorname{Pr}=v / \alpha(\alpha$ : thermal conductivity), respectively.

Figure 1 shows the computational volume and coordinate systems. The computational volume is the rectangular box. The origin of the axes was set at the center of the jet. The wall-normal direction, $y$ and two horizontal directions, $x$ and $z$ were set; and the velocity component for each direction is denoted as $u, v$, and $w$, correspondingly.

Spatial discretization was performed with a sine or cosine series expansion in the $x$ - and $z$-directions and a sixth-order compact scheme (Lele, 1992) in the $y$-direction. The velocity components were discretized as follows such that the boundary conditions were satisfied: 


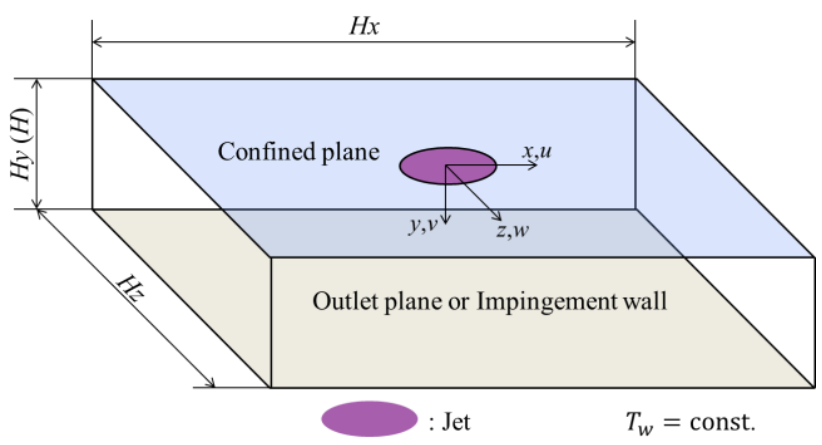

Fig. 1 Coordinate system and computational domain
Table 1 Calculation conditions

\begin{tabular}{|c|c|}
\hline $\begin{array}{c}\text { Domain } \\
(H x \times H y \times H z)\end{array}$ & $\begin{array}{c}7 D \times 15 D \times 7 D \text { (Free jet) } \\
7 D \times(4-10) D \times 7 D \text { (Impingement) }\end{array}$ \\
\hline Grid number & $\begin{array}{c}128 \times 150 \times 128 \text { (Free jet) } \\
128 \times 80-200 \times 128 \text { (Impingement) }\end{array}$ \\
\hline Reynolds Number & $\mathrm{Re}=V_{0} D / v=1500$ \\
\hline Prandtl Number & $\mathrm{Pr}=0.707$ \\
\hline Strouhal Number & $\mathrm{St}_{a}=f_{a} D / V_{0}=0.45$ \\
\hline Frequency ratio & $R_{f}=2.4,2.5,2.6$ \\
\hline Excitation amplitude & $A_{a}=A_{h}=15 \%$ \\
\hline Impingement distance & $H=4 D, 6 D, 8 D, 10 D$ \\
\hline
\end{tabular}

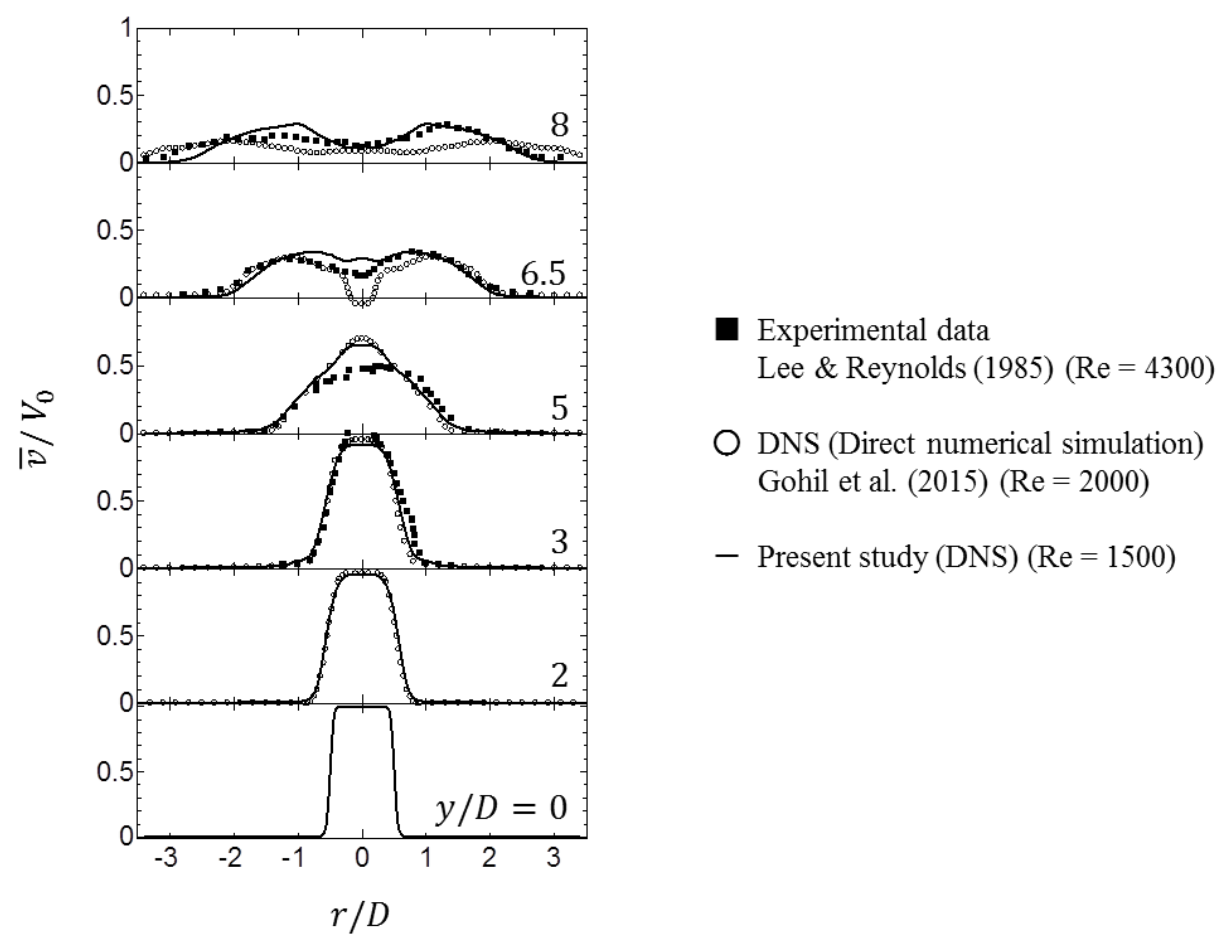

Fig. 2 Time-averaged streamwise velocity profiles of a blooming free jet

$$
\begin{aligned}
& u(x, y, z)=\sum u_{m n}(y) \cos \left(k_{m} x\right) \sin \left(k_{n} z\right) \\
& v(x, y, z)=\sum v_{m n}(y) \sin \left(k_{m} x\right) \sin \left(k_{n} z\right) \\
& w(x, y, z)=\sum w_{m n}(y) \sin \left(k_{m} x\right) \cos \left(k_{n} z\right)
\end{aligned}
$$

To remove the numerical instability due to the nonlinear terms, the 2/3-rule (truncating 1/3 of the large wave numbers) was applied for the horizontal directions, and an implicit filtering for the wall-normal direction was conducted with the sixth-order compact scheme. For time advancement, the third-order Adams-Bashforth method was used. The well-known MAC method was employed for pressure-velocity coupling, which resulted in a Poisson equation for the pressure. After the Poisson equation was expanded with a sine series in the $x$ - and $z$-directions, independent differential equations were obtained for each wave number; subsequently, they were discretized with the sixth-order compact scheme. Finally, a pentadiagonal matrix was deduced for each wave number.

In the present simulation code, these matrices were solved using the LU decomposition method. When vortical structures approached the side boundary of the impingement wall, relatively strong low-pressure regions formed by the vortical structures did not satisfy the pressure boundary condition, i.e., $p=0$. In the present simulation, because the 
spectral method was used, the occurrence of this discrepancy at the side boundary induced unphysical numerical oscillations in the entire flow field. Hence, the vortical motions should be artificially reduced near the boundary. In the present simulation, a fringe (buffer) region (Nordstrom et al., 1999) was introduced near the outflow boundary to reduce perturbations using an appropriate external force. A convective condition was introduced for both momentum and energy equations by solving the simplified convective equations, with $U_{c}$ the convective velocity, which is $\mathrm{x}$, z-averaged mean velocity in the outlet plane, at every time step, for free jets (Orlanski, 1976):

$$
\frac{\partial u_{i}}{\partial t}+U_{c} \frac{\partial u_{i}}{\partial y}=0, \frac{\partial T}{\partial t}+U_{c} \frac{\partial T}{\partial y}=0
$$

\subsection{Calculation conditions}

At the inflow boundary, a hyperbolic tangent profile, typically known as a top-hat profile, was prescribed across the jet inlet of diameter $D$ for the axial velocity:

$$
V_{m}(r)=\frac{V_{0}}{2}-\frac{V_{0}}{2} \tanh \left[\frac{1}{4} \frac{R}{\theta_{0}}\left(\frac{r}{R}-\frac{R}{r}\right)\right]
$$

where, $V_{0}$ is the center velocity. The symbol $\theta_{0}$, which denotes the initial momentum thickness, is set as $\theta_{0} / R=0.05$ and $R(=D / 2)$ is the radius of the inlet jet. The symbol $r$ measures the radial distance from the centerline of the jet. The inlet velocity profile is determined by referring to a previous study (Silva and Metais, 2002). The hyperbolic tangent profile, as expressed by Eq. (6), approximates the top-hat inflow velocity profile typically observed in jet experiments and was shown to generate a more realistic potential core length and turbulent transition. In the equations above, a random perturbation, $\left(u_{\text {rad }}, v_{\text {rad }}, w_{\text {rad }}\right)$ with $1 \%$ of the strength of $V_{0}$ is added to the inflow velocity.

Subsequently, we demonstrate the inflow velocity of the blooming jet based on the studies of Gohil et al. (2015) and Tyliszczak (2018).

$$
\frac{V(r, t)}{V_{m}(r)}=1+A_{a} \sin \left(2 \pi f_{a} t\right)+A_{h} \sin \left[2 \pi f_{h} t+\theta_{c}\right] \sin (\pi r / D)
$$

where the symbols $f_{a}$ and $f_{h}$ denote frequencies of the axial and helical excitation, respectively, which are defined by the Strouhal number $\mathrm{St}_{\mathrm{a}}\left(=f_{a} D / V_{0}=0.45\right)$; the frequency ratio $R_{f}=f_{a} / f_{h}=2.4-2.6$. It has been reported that blooming jets spread considerably in this $R_{f}$ range (Tyliszczak, 2015). The excitation strength of the axial and helical modes is $A_{a}=A_{h}=15 \%$, and $\theta_{c}$ is the azimuthal angle. The computational domain is set to a rectangular domain of $7 D \times 15 D \times 7 D$ for the free jets and $7 D \times(4-10) D \times 7 D$ for the impinging jets. In addition, the computational grid number used in this simulation is $\left(N_{x}, N_{y}, N_{z}\right)=(128,150,128)$ for the free jets and $(128,80-200,128)$ for the impinging jets, in which $N_{y}$ is varied with the change in the impingement distance. For the blooming free jets, the inflow temperature profile is implemented in a manner similar to that of the inflow velocity profile:

$$
T=\frac{T_{0}}{2}-\frac{T_{0}}{2} \tanh \left[\frac{1}{4} \frac{R}{\theta_{0}}\left(\frac{r}{R}-\frac{R}{r}\right)\right]
$$

where the inflow temperature, $T_{0}$ is assumed to be higher than the ambient temperature, $T_{a}$ (i.e., $T_{0}>T_{a}$ ). For the blooming impinging jets, the inflow temperature, $T_{0}$ and the ambient temperature, $T_{a}$ are assumed to be higher than the wall temperature, $T_{w}$, i.e., $T_{0}=T_{a}>T_{w}$.

The statistical properties are averaged over time, and the mean quantity is denoted with a bar. The parameters for the present calculations are shown in Table 1.

\section{Results and discussion}

\subsection{Blooming free jet (BFJ)}

Figure 2 shows a comparison of the time-averaged velocity profiles of the BFJ with the experimental results by Lee and Reynolds (1985) and the numerical results by Gohil et al. (2015). In the experiment, the excitation frequency was 0.46, the frequency ratio 2.4, and the Reynolds number 4300. In Gohil's study, the excitation frequency was 0.5 , the frequency ratio 2.4, and the Reynolds number 2000. In the present study, $\mathrm{St}_{\mathrm{a}}=0.45$ and the Reynolds number was 


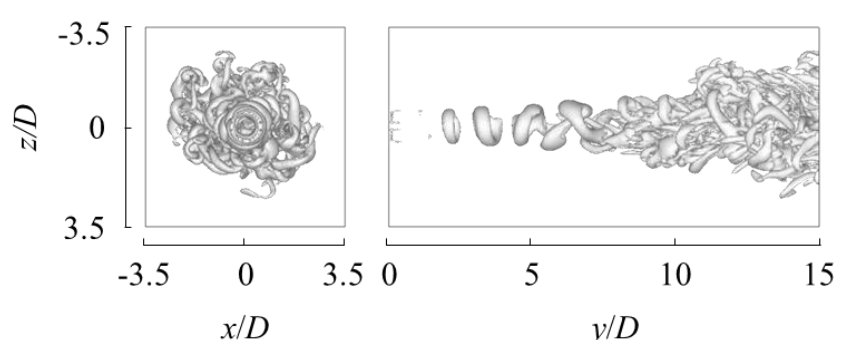

(a) Free jet

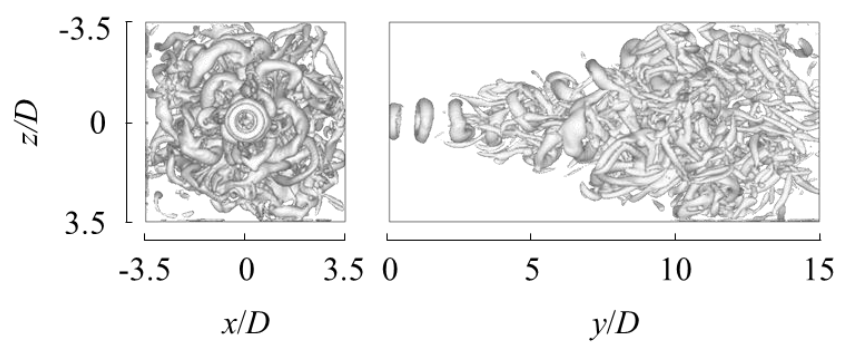

(b) $R_{f}=2.4$
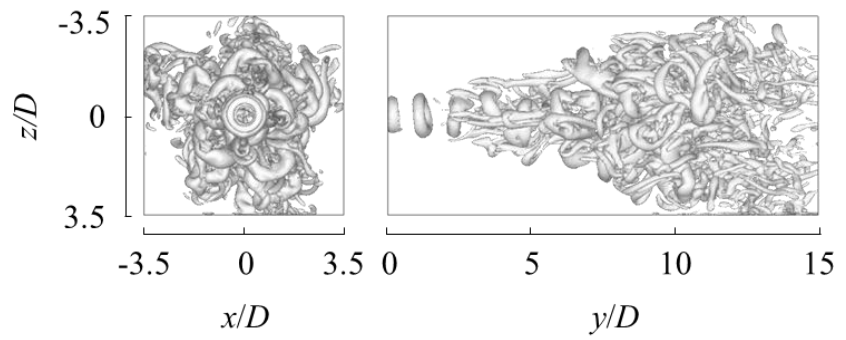

(c) $R_{f}=2.5$
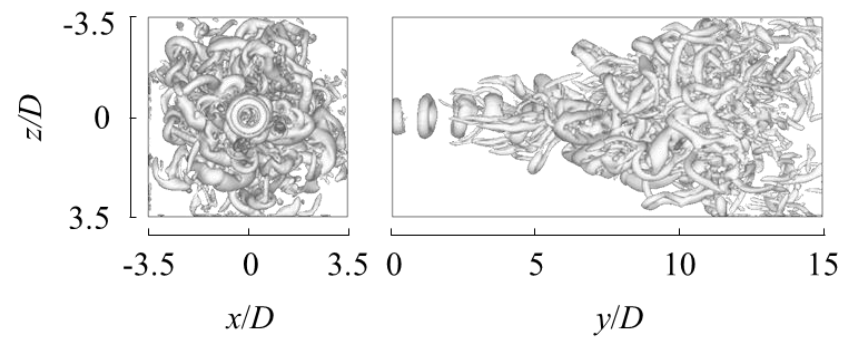

(d) $R_{f}=2.6$

Fig. 3 Instantaneous vortex structures $(Q=0.1)$

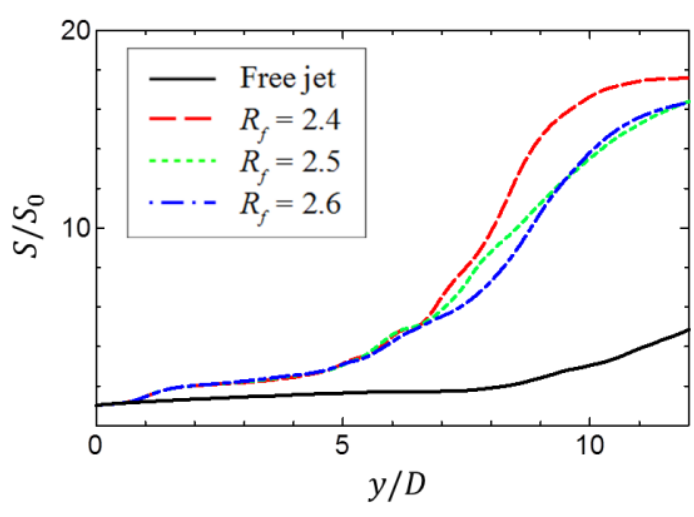

Fig. 4 Streamwise mean entropy distribution

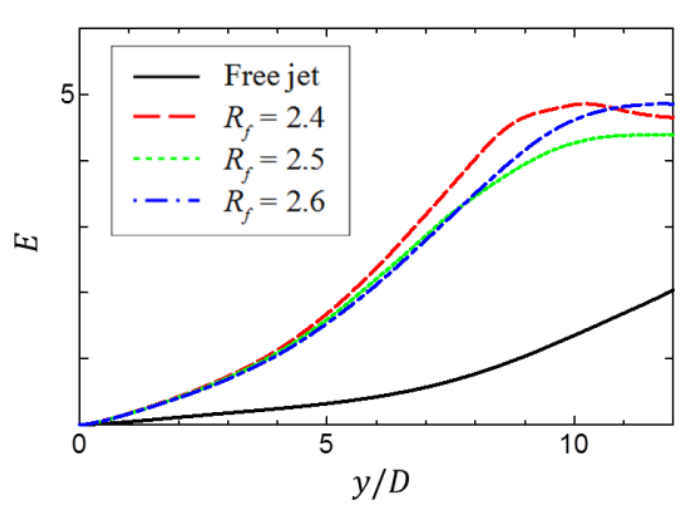

Fig. 5 Streamwise distribution of entrainment

1500, although the frequency ratio was 2.4. Despite the different Reynolds number, Fig. 2 reveals a good agreement between the present study and the field data; the velocity magnitude and the manner of velocity distribution agreed well. At $y / D=0$, the time-averaged velocity distribution indicated a top-hat profile. Until $y / D=5$, the time-averaged velocity profile did not exhibit any jet splitting. However, at $y / D=6.5$, two peaks were observed, demonstrating that jet splitting occurred. At $y / D=8$, the velocity distribution became flat and the two peaks shifted outward, indicating the strong spreading of the blooming jet in the radial direction. Meanwhile, until $y / D=5$, our and Gohil's results agreed well. However, at $y / D=6.5$, the difference in the centerline velocity increased. At $y / D=8$, our and Gohil's results did not agree well, whereas our result and the experimental data matched better compared with Gohil's results.

Figure 3 shows the instantaneous vortex structures of the blooming jets with each frequency ratio. In the present study, the Q-criterion was used, where $\mathrm{Q}=(\Omega \mathrm{ij}-\mathrm{Sij} \mathrm{Sij}) / 2$, with $\Omega \mathrm{ij}$ and $\mathrm{Sij}$ are the rotation and deformation tensors, respectively, which are associated with the second invariant of the velocity gradient tensor. It is visualized perpendicular to and straight from the jet axis to extract the dominant vortical structures from the simulation data to study the effect of the frequency ratio on the vortical structures. Positive values of $\mathrm{Q}$ indicate regions where rotation is dominant and serves as a good indicator of coherent vortices in turbulent flows (Hunt et al., 1988). In the uncontrolled 
free jet, vortical structures formed in the near field broke down and spread radially; subsequently, it propagated toward the far field. However, these traits changed significantly with the corresponding frequency ratio. For the case with $R_{f}=$ 2.5, the effect of the axial excitation was evident as toroidal vortex rings were clearly observed in the near field. These toroidal rings began to break down gradually; subsequently, it propagated toward the far field. In particular, it was observed that the jet split into five directions, as five separate azimuthal locations existed, in which the maxima of the axial excitation and the maxima of the helical excitation occurred simultaneously in 2 helical cycles. For the cases with $R_{f}=2.4$ and 2.6, the spreading of the jet was slightly large at $R_{f}=2.4$. Similar to that observed at $R_{f}=2.5$, toroidal vortex rings were clearly observed in the near field and began to break down gradually; subsequently, it propagated toward the far field. The vortical structures distributed in a relatively uniform manner, whereas the blooming jet at $R_{f}=$ 2.5 split into five directions. In addition, for cases with $R_{f}=2.4$ and 2.6, 12 or 13 separate azimuthal locations existed, in which the maxima of the axial excitation and the maxima of the helical excitation occurred simultaneously in 5 helical cycles. Hence, because the jets split into 12 or 13 azimuthal directions, the jets appeared to spread evenly. These quantitative characteristics agreed well with those reported in previous studies (Gohil et al., 2015) (Tyliszczak, 2018).

Owing the instantaneous structures of the jets, the flow pattern of the blooming jets changed significantly toward the far field, and an exceptional radial expansion effect could be achieved. However, because it was difficult to validate the mixing state of the jets using only the current evaluation method, the mixing state must be evaluated quantitatively. In particular, we investigated compound jets (Tsujimoto et al., 2006) and discovered that the mixing efficiency could not be determined using the simple measure that had been developed for axisymmetric jets. Therefore, we investigated the appropriate measure for quantifying the mixing efficiency and discovered statistical entropy to be a good measure (Tsujimoto et al., 2009).

Statistical entropy based on passive scalar concentration was proposed by Everson et al. (1998). They demonstrated the characteristics of this measure by examining experimental data. In the following, we provide a simple explanation for this measure. Boltzmann proposed the statistical entropy, which is defined as the logarithm of the combination, $W$.

$$
S=k \ln W
$$

where $k$ is the Boltzmann constant; $W$ is the combination of the number of molecules in the $i$-th coarse-grained cell, $N i$, i.e., $W=\frac{N !}{N_{1} ! N_{2} ! \cdots N_{M} !}$. Because an incompressible flow is assumed in the present study, the temperature can be correlated with the concentration of the passive scalar, i.e., $\phi=\left(T-T_{a}\right) /\left(T_{0}-T_{a}\right)$. Considering the small volume surrounding a grid point $i, \Delta V\left(=\Delta_{x} \Delta_{y} \Delta_{z}\right)$, the number of molecules is denoted by $N_{i}=\phi_{\mathrm{i}} \Delta V$; therefore,

$$
S=k \Delta V\left[\Phi \ln \Phi-\sum_{i=1}^{M} \phi_{\mathrm{i}} \ln \phi_{\mathrm{i}}\right]
$$

where $\Phi=\Sigma \phi_{\mathrm{i}}$.

To evaluate the mean mixing characteristics quantitatively, Fig. 4 shows the distribution of the time-averaged streamwise entropy $S / S_{0}$, which is defined in Eq. (10). Regarding the mean mixing characteristics of the uncontrolled jet, beyond the region where the potential core broke down, $y / D=8$, the value increased gradually and the mixing became active owing to the spreading of the jet. For the blooming jets, although the instantaneous vortical structures were slightly different, the same elevation continued until $y / D=5$ for each $R_{f}$; subsequently, the mixing became active gradually at the region beyond that. Conclusively, the blooming jets demonstrated significantly better mixing performances than the uncontrolled jet.

Additionally, to evaluate the entrainment properties numerically, the entrainment $E$ is used. The entrainment $E$ is defined as follows:

$$
E=\frac{\iint V d x d z-Q_{0}}{Q_{0}}
$$

which is normalized by the inlet volumetric flow rate $Q_{0}$; the result is shown in Fig. 5. For the uncontrolled jet, the entrainment increased gradually toward the downstream. Unlike the uncontrolled jet, a strong entrainment occurred in the upstream region of the blooming jets; the entrainment increased gradually until $y / D=10$, however, it saturated beyond $y / D=10$. Conclusively, the blooming jets exhibited greater entrainment than the uncontrolled jet. 


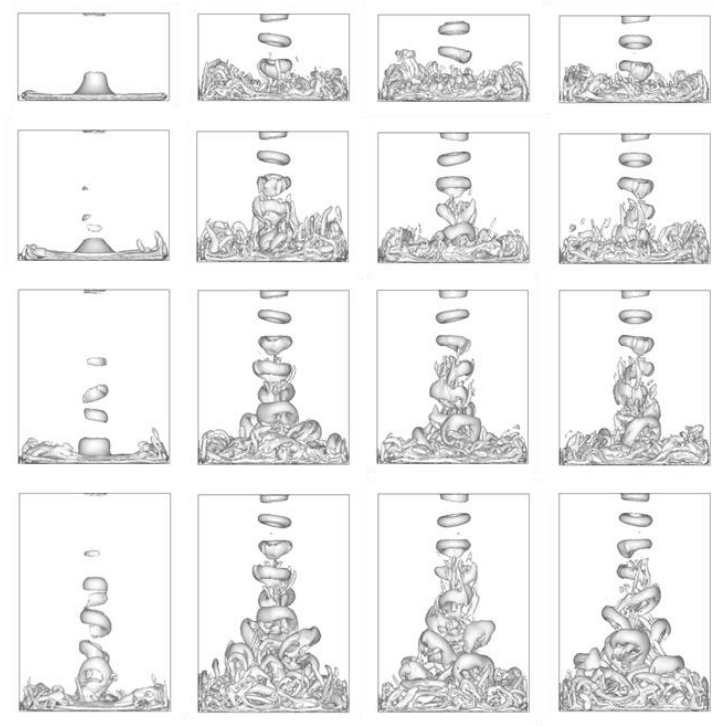

(a) Side view
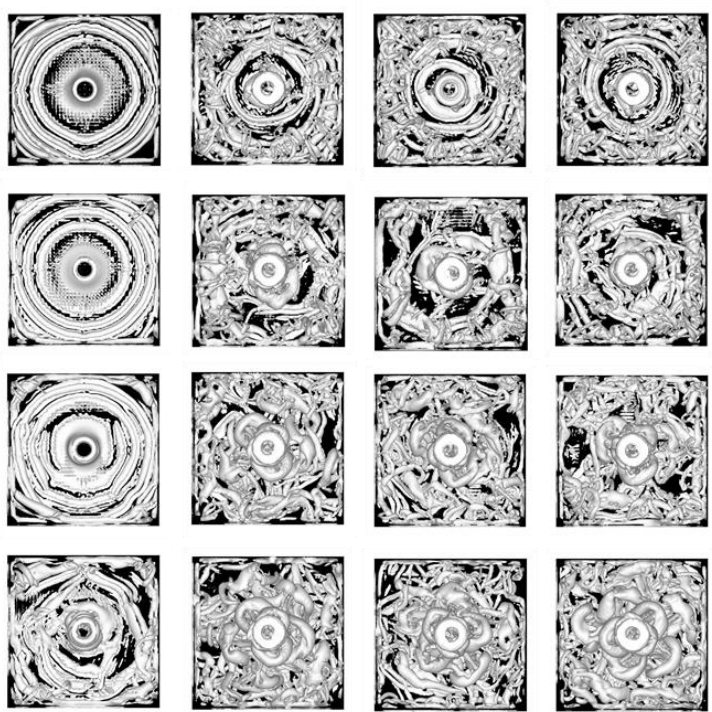

(b) Top view

Fig. 6 Instantaneous vortex structures $(Q=0.1)$, first column: CIJ, second: BIJ $\left(R_{f}=2.4\right)$, third: BIJ $\left(R_{f}=2.5\right)$, fourth: BIJ $\left(R_{f}=2.6\right)$; top to bottom row: $H=4 D, 6 D, 8 D$, and $10 D$.

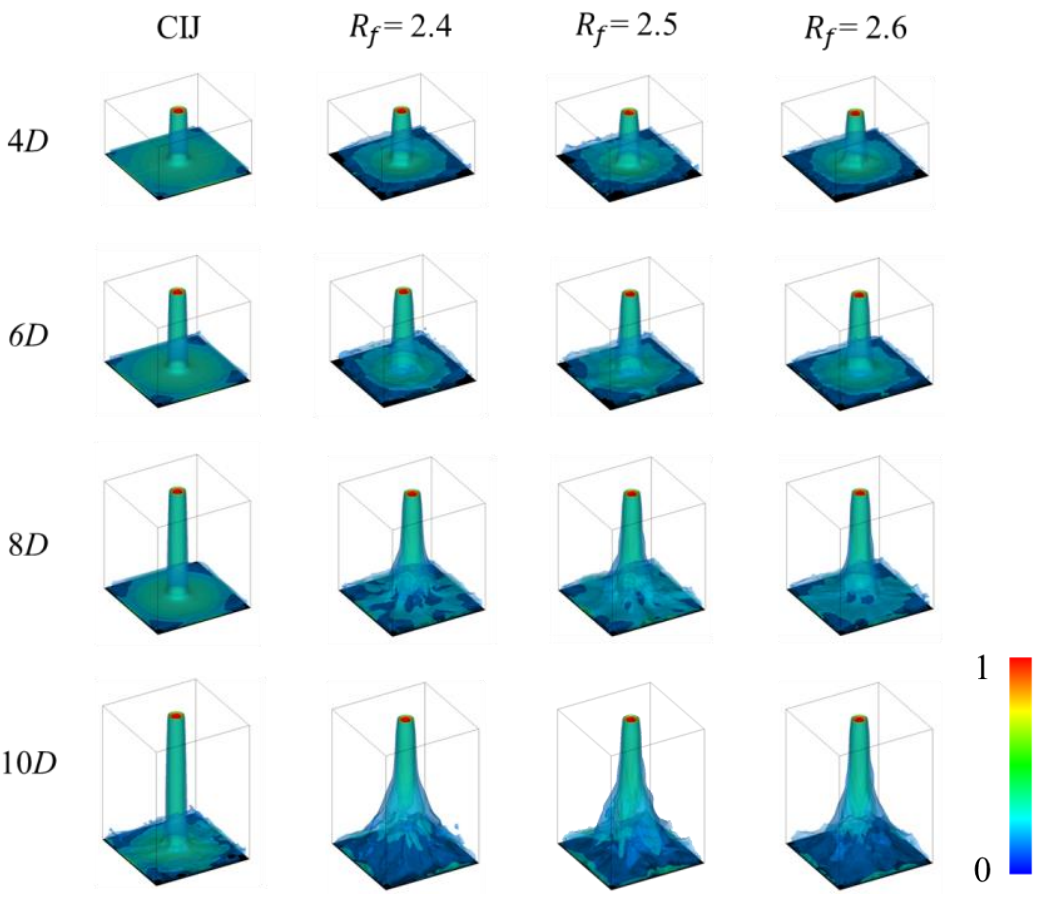

Fig. 7 Contours of the mean velocity magnitude, first column: CIJ, second: BIJ $\left(R_{f}=2.4\right)$, third: BIJ $\left(R_{f}=2.5\right)$, fourth: BIJ $\left(R_{f}=2.6\right)$; top to bottom row: $H=4 D, 6 D, 8 D$, and $10 D$.

\subsection{Blooming impinging jet (BIJ)}

Instantaneous vortical structures are visualized in Fig. 6 in the same manner as those shown in Fig. 3. As shown in Fig. 6(a) for the case of the uncontrolled conventional impinging jet (CIJ), the formation of the vortex structures became more active as the impingement distance increased. Meanwhile, in the case of the BIJ, toroidal vortex rings were formed from the vicinities of the inflow as with $\mathrm{BFJ}$, regardless of the impingement distance. As the impingement distance increased, the toroidal vortex rings interfered with each other and spread more in the radial direction. In 
addition, compared with the BFJ in Fig. 3, it was observed that the expansion in the direction perpendicular to the jet axis was suppressed owing to the impingement wall in the BIJ. The formation of vortex structures near the wall weakened, and the induced velocity due to the toroidal vortex rings reduced, resulting in the suppression of BIJ spreading. It is noteworthy that the significant structural change due to the difference in the frequency ratio was not observed in Fig. 6(a). On the contrary, a difference in the formation of vortex structures on the impingement wall was observed, as shown in Fig. 6(b). In the CIJ, when the impingement distance was short, large concentric vortices were formed on the impingement wall. As the impingement distance increased, the formation of the vortex structures above the impingement wall enhanced; hence, the concentric structures disappeared. Meanwhile, in the BIJ, in which the impingement distance was small, concentric structures were observed, and the large concentric structure disappeared as the impingement distance increased, as with CIJ. To observe the state of the flow field more, isosurfaces of the time-averaged velocity contours are shown in Fig. 7. In the CIJ, the issuing jet did not diffuse toward the impingement wall regardless of the impingement distance. In the BIJ, the velocity distribution varied significantly according to the impingement distance; when the impingement distance was larger than $8 D$, the jet spread significantly toward the downstream. It is noteworthy that the lower the $R_{f}$, the more the jet spread.

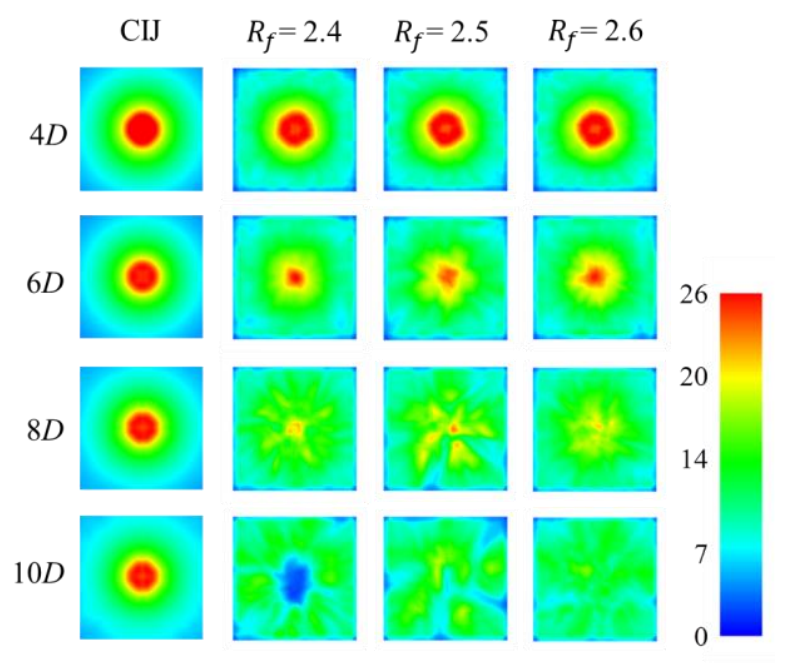

Fig. 8 Contours of the mean local Nusselt number, first column: CIJ, second: BIJ $\left(R_{f}=2.4\right)$, third: BIJ $\left(R_{f}=2.5\right)$, fourth: BIJ $\left(R_{f}=2.6\right)$; top to bottom row: $H=4 D, 6 D, 8 D$, and $10 D$.

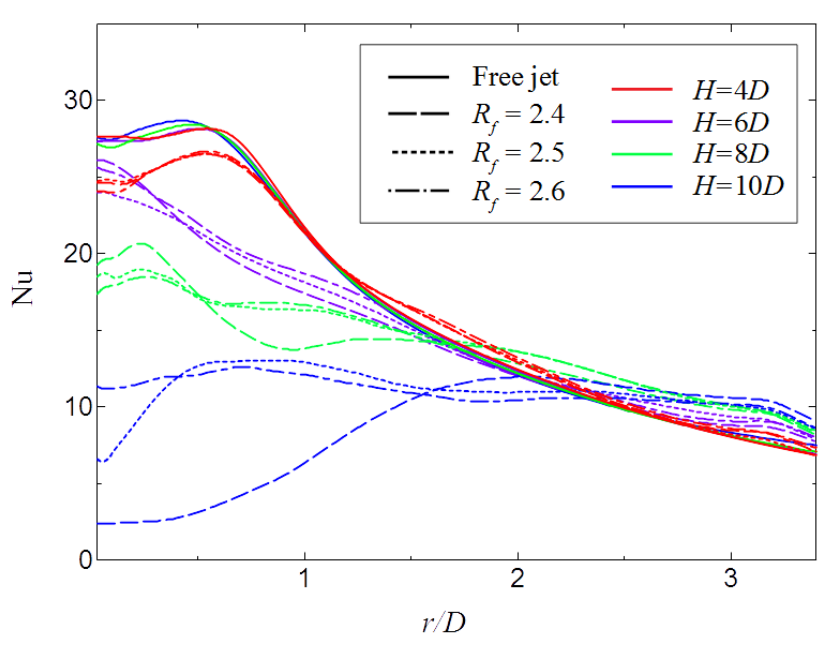

Fig. 9 Distribution of the mean Nusselt number

To evaluate the heat transfer characteristics qualitatively, contours of the time-averaged local Nusselt number are shown in Fig. 8. In the CIJ, a high heat transfer appeared around the impingement point, as indicated by the region in red, regardless of the impingement distance. Furthermore, in the BIJ, when the impingement distance was smaller than $6 D$, high heat transfer characteristics were demonstrated around the impingement point, as with CIJ. However, based on the mean velocity distribution, if the impingement distance was longer than $8 D$, the local high heat transfer characteristics would disappear and relatively uniform heat transfer characteristics would be obtained. The heat transfer proceeded more uniformly when the impingement distance was $10 D$. For the case with $R_{f}=2.5$, corresponding to the characteristics of the flow structure, five branches were observed. For cases with $R_{f}=2.4$ and 2.6, the state of the branch became ambiguous, and the heat transfer proceeded more uniformly. In particular, for the case with $R_{f}=2.4$, the heat transfer around the impingement point reduced significantly owing to the strong splitting of the jets, as shown in Fig. 7. Additionally, to evaluate the heat transfer characteristics quantitatively, the distribution of the mean Nusselt number is shown in Fig. 9. In the CIJ, the Nusselt number distribution did not change significantly based on the impingement distance. On the contrary, in the BIJ, the distribution of the Nusselt number varied significantly based on the impingement distance, and the effect of the frequency ratio on the Nusselt number became more evident as the impingement distance increased. 


\section{Conclusions}

In the present study, we conducted a DNS of blooming jets arranged at the inflow of a flow field to demonstrate the flow and heat characteristics of impinging jets controlled using blooming excitations.

1. After the jet was issued from the inlet, toroidal vortex rings were formed in the near field, and the interference between each individual vortex ring caused a strong jet spread on the impingement wall. The structure of the CIJ did not change significantly within the parameter range of the impingement distance; however, for the BIJ, the impingement distance significantly affected the jet spreading on the impingement wall.

2. For the CIJ, the Nusselt number on the impingement wall exhibited a peak around the impingement point regardless of the impingement distance; subsequently, it decreased as the distance from the impingement point increased. Meanwhile, for the BIJ, the heat transfer characteristics on the impingement wall modulated significantly according to the change in the flow structure based on the impingement distance.

3. When the impingement distance was small, the effect of the frequency ratio on the Nusselt number was slight, and the Nusselt number decreased monotonically as the distance from the impingement point increased. However, as the impingement distance increased, the effect of the frequency ratio on the Nusselt number became prominent. When the frequency ratio was small, the Nusselt number decreased significantly around the impingement point, whereas when the frequency ratio was large, a substantially uniform distribution of the Nusselt number was achieved on the wall.

\section{References}

Canuto, C., Hussaini, M. Y., Quarteroni, A., Zang, T. A., Spectral Methods in Fluid Dynamics, Springer (1988).

Dairay, T., Fortune, V., Lamballais, E. and Brizzi, L.E., LES of a turbulent jet impinging on a heated wall using high-order numerical schemes, Int. J. Heat Fluid Flow, Vol.50 (2014), pp.177-187.

Dairay, T. Fortune, V., Lamballais, E. and Brizzi, L.E., Direct numerical simulation of a turbulent jet impinging on a heated wall, J. Fluid Mech., Vol.764 (2015), pp.362-394.

Danaila, I., Boersma, B.J., Direct numerical simulation of bifurcating jets, Phys. Fluids, Vol.12 (2000), pp.1255-1257.

Everson, R., Manin, D., Sirovich, L. and Winter, M., Quantification of mixing and mixing rate from experimental observations, AIAA J., Vol.36, No.2 (1998), pp.121-127.

Freund, J.B., Moin, P., Jet mixing enhancement by high amplitude fluidic actuation, AIAA J., Vol.38, No.10 (2000), pp. $1863-1870$.

Gohil, T.B., Saha, A.K., Muralidhar, K., Control of flow in forced jets: a comparison of round and square cross sections, J. Visual., Vol. 13 (2010), pp.141-149.

Gohil, T.B., Saha, A.K., Muralidhar, K., Direct numerical simulation of free and forced square jets, Int. J. Heat Fluid Flow, Vol.52 (2015), pp.169-184.

Gohil, T. B., Saha, A.K. and Muralidhar, K., Simulation of the blooming phenomenon in forced circular jets, J. Fluid Mech., Vol.783 (2015), pp.567-604.

Hadžiabdić, M. and Hanjalić, K., Vortical structures and heat transfer in a round impinging jet, J. Fluid Mech., Vol.596 (2007), pp.221-260.

Hattori, H. and Nagano, Y., Direct numerical simulation of turbulent heat transfer in plane impinging jet, Int. J. Heat Fluid Flow, Vol.25 (2004), pp.749-758.

Hilgers, A. and Boersma, B. J., Optimization of turbulent jet mixing, Fluid Dyn. Res., Vol.29, No.6 (2001), pp. $345-368$.

Hunt, J. C. R., Wray, A. A., Moin, P., Eddies, streams, and convergence zones in turbulent flows, Center for Turbulence Research Report CTR-S88 (1988).

Jambunathan, K., Lai, E., Moss, M.A. and Button, B.L., A review of heat transfer data for single circular jet impingement, Int. J. Heat Fluid Flow, Vol.13 (1992), pp.106-115. 
Kataoka, K., Impingement heat transfer augmentation due to large scale eddies, In Heat Transfer 1990, Proc. 9th Intl Heat Transfer Conf., Vol.1 (1990), pp.255-273.

Lee, M. and Reynolds, W.C., Bifurcating and blooming jets, Tech. Rep., TF-22, Stanford Univ., (1985)

Lele, S. K., Compact finite difference schemes with spectral-like resolution, J. Comput. Phys., Vol.103 (1992), pp.16-42.

Nordstrom, J., Nordin, N., and Henningson, D. S., The fringe region technique and the fourier method used in the direct numerical simulation of spatially evolving viscous flows, SIAM J. Sci. Comp., Vol.20 (1999), pp.1365-1393.

Orlanski, I., A simple boundary condition for unbounded hyperbolic flows, J. Comput. Phys, Vol.21 (1976), pp. 251-269.

Reynolds, W.C., Parekh, D.E., Juvet, P.J.D. and Lee, M.J.D., Bifurcating and blooming Jets, Annu. Rev. Fluid Mech., Vol.35 (2003), pp. 295-315.

Satake, S. and Kunugi, T., Direct numerical simulation of an impinging jet into parallel disks, International Journal of Numerical Methods for Heat and Fluid Flow, Vol.8 No.7 (1998), pp.768-780.

Silva, C. B. and Metais, O., Vortex control of bifurcating jets: A numerical study, Phys. Fluids, Vol.14 (2002), pp. 3798-3819.

Tsubokura, M., Kobayashi, T., Taniguchi, N. and Jones, W. P., A numerical study on the eddy structure of impinging jets excited at the inlet, Int. J. Heat Fluid Flow, Vol.24 (2003), pp.500-511.

Tsujimoto, K., Shakouchi, T. Sasazaki S and Ando, T., Direct Numerical Simulation of Jet Mixing Control Using Combined Jets, JSME Int. J. Ser. B, Vol.49 (2006), pp. 966-973.

Tsujimoto, K., Kariya, S., Shakouchi, T. and Ando, T., Investigation on Jet Mixing Rate Based on DNS of Excitation Jets, Trans. JSME, Vol.74 (2008), pp.34-41.

Tsujimoto, K., Kariya, S., Shakouchi, T. and Ando, T., Evaluation of jet mixing rate based on DNS data of excitation jets, Int. J. Flow Control, Vol.1 (2009), pp. 213-225.

Tyliszczak, A., Boguslawski, A., LES of variable density bifurcating jets, Complex Effects in Large Eddy Simulations. No. 56 in Lecture Notes in Computational Science and Engineering, Springer (2007), pp. 273-288.

Tyliszczak, A., Geurts, B.J., Parametric analysis of excited round jets - numerical study, Flow, Turbul. Combust., Vol.93, No.2 (2014), pp. 221-247.

Tyliszczak, A., Geurts, B.J., Controlled mixing enhancement in turbulent rectangular jets responding to periodically forced inflow conditions, J. Turbul., Vol.16 (2015), pp.742-771.

Tyliszczak, A., Multi-armed jets: a subset of the blooming jets. Phys. Fluids, Vol.27 (2015), pp. 1-7, 041703.

Tyliszczak, A., Parametric study of multi-armed jets, Int. J. Heat Fluid Flow, Vol.73 (2018), pp.82-100.

Viskanta, R., Heat transfer to impinging isothermal gas and flame jets, Exp. Therm. Fluid Sci., Vol.6 (1993), pp.111-134. 